\title{
Determination of the solar rotation parameters via orthogonal polynomials
}

\author{
T.G. Mdzinarishvili a,b , B.M. Shergelashvili ${ }^{\text {b,c,d,*}}$, D.R. Japaridze ${ }^{a, b}$, B.B. Chargeishvili ${ }^{\text {a,b }}$ \\ A.G. Kosovichev ${ }^{\mathrm{e}}$, S. Poedts ${ }^{\mathrm{a}}$ \\ ${ }^{a}$ Center for Mathematical Plasma Astrophysics, Department of Mathematics, KU Leuven, 200 B, B-3001 Leuven, Belgium \\ ${ }^{\mathrm{b}}$ Abastumani Astrophysical Observatory at Ilia State University, University St. 2, Tbilisi, Georgia

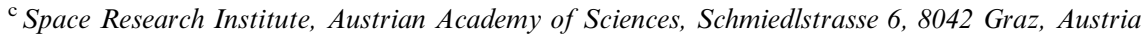 \\ ${ }^{\mathrm{d}}$ Combinatorial Optimization and Decision Support, KU Leuven campus Kortrijk, E. Sabbelaan 53, 8500 Kortrijk, Belgium \\ ${ }^{\mathrm{e}}$ New Jersey Institute of Technology, Newark, NJ 07103, USA
}

Received 12 September 2019; received in revised form 4 January 2020; accepted 13 January 2020

\begin{abstract}
Accurate measurements of the solar differential rotation parameters are necessary for understanding the solar dynamo mechanism. We use the orthogonalization process to estimate these parameters. The advantage of the orthogonalization of the data in the tracer motion statistical analysis is outlined. The differential rotation is represented in terms of various types of polynomials. We compare the quality of a set of models of the solar differential rotation using the Akaike information criterion and choose the best one. Applying the proposed method, we studied the solar differential rotation and its North-South asymmetry using observations of coronal holes. A statistical analysis of observations from the Atmospheric Imaging Assembly (AIA) on Solar Dynamics Observatory (SDO) reveals the differential rotation pattern of coronal holes and its North-South asymmetry.
\end{abstract}

(C) 2020 COSPAR. Published by Elsevier Ltd. All rights reserved.

Keywords: Statistical data analysis; Solar rotation; Solar coronal holes

\section{Introduction}

The solar rotation velocity changes with both latitude and radius. This differential rotation is supposed to play a key role in the solar dynamo mechanism. Due to its radial dependence, it also has an impact on the internal structure of the Sun caused by instabilities related to rotational shear layers. It is assumed that the solar dynamo is controlled by global convection and differential rotation, as well as the topological deformation of the magnetic field lines in the solar convective zone (Yoshimura, 1996).

\footnotetext{
* Corresponding author at: Abastumani Astrophysical Observatory at Ilia State University, University St. 2, Tbilisi, Georgia.

E-mail address: bidzina.shergelashvili@iliauni.edu.ge (B.M. Shergelashvili).
}

There are two methods for measuring the surface rotation velocities: spectroscopy and tracer motion. Using the spectroscopic method, the Doppler line shift is measured. This provides the angular velocity in those layers of the solar atmosphere where the spectral lines chosen for the measurements are formed. The tracer motion method, on the other hand, involves either the tracking of the rotation rates of individual resolved features on the surface (such as sunspots, active regions, super-granular cells, etc.) as they pass across the disk, or the cross-correlation of magnetograms and Dopplergrams obtained at different times. The solar rotation velocity along the radius inside the Sun is determined by applying helioseismology (Schou et al., 1998; Beck, 2000; Paternò, 2010). 
The angular velocity $\omega$ of the surface differential rotation is thus a function of the solar latitude, $\theta$. Historically, it is given in terms of even order trigonometric functions (Newton and Nunn, 1951; Howard and Harvey, 1970) which automatically implies that there is no asymmetry between the two hemispheres (both hemispheres obey the same differential rotational law), viz.

$\omega(\theta)=A+B \sin ^{2} \theta+C \sin ^{4} \theta, \quad-90^{\circ} \leqslant \theta \leq 90^{\circ}$.

Here, the coefficient $A$ provides the equatorial rotation rate $\left(\theta=0^{\circ}\right)$, and the coefficients $B$ and $C$ describe the differential rotation.

Eq. (1) can be written as a polynomial:

$y(x)=A+B x^{2}+C x^{4}, \quad-1 \leqslant x \leq 1$,

where $x=\sin \theta$, and thus $y(x)=\omega(\sin \theta), \quad-90^{\circ} \leqslant$ $\theta \leq 90^{\circ}$.

It should be noted that in expansion (2) the basis functions (vectors),

$v_{1}=v_{1}(x)=1, \quad v_{2}=v_{2}(x)=x^{2}, \quad v_{3}=v_{3}(x)=x^{4}$,

are not orthogonal (Lipschutz and Lipson, 2009). Clearly, Eq. (2) can also be rewritten in terms of these basis functions as follows

$y(x)=A v_{1}(x)+B v_{2}(x)+C v_{3}(x), \quad-1 \leqslant x \leq 1$.

Upon applying this formula to fit data, the nonorthogonality of the basis functions results in correlated errors ('cross-talk') among the estimated coefficients $A, B$ and $C$ (in fitting procedure, it is assumed that we know exact values of $x$ ). In general, the polynomial fitting procedure will yield values for the coefficients that depend on the degree of the polynomial fitted to the data. Therefore, to overcome this problem a set of continuous Gegenbauer orthogonal polynomials has been used for measuring the solar surface rotation velocities (Snodgrass and Howard, 1985; Snodgrass and Ulrich, 1990; Gigolashvili et al., 2003, 2005, 2007, 2011, 2013; Javaraiah, 2011; Sudar et al., 2015) as well as for determining the internal differential rotation by helioseismology (Kosovichev, 1988).

In this case, instead of Eq. (2) we have the equation

$$
\begin{aligned}
y(x)= & A_{G}+B_{G}\left(5 x^{2}-1\right)+C_{G}\left(21 x^{4}-14 x^{2}+1\right), \\
& -1 \leqslant x \leq 1 .
\end{aligned}
$$

Here $1,\left(5 x^{2}-1\right)$ and $\left(21 x^{4}-14 x^{2}+1\right)$ are the Gegenbauer orthogonal polynomials with weights defined in Snodgrass and Ulrich (1990). The coefficients $A_{G}, B_{G}$ and $C_{G}$ are then estimated from observational data using a regression function (5).

It should be noted that when using continuous orthogonal polynomials, we cannot completely overcome the 'cross-talking' among the coefficients in polynomial regression. As a matter of fact, the cross-talk remains because of the discreteness of the observational data sample.
In particular, the results depend on the uniformity and the density of covering the entire range of the independent variable $x$ using observational data (Bevington and Robinson, 2003). However, it is well-known that sunspot observations cover low latitude areas more densely than the high latitude ones. In this case, uniformity of observational data is thus difficult to achieve and this is also true for other solar rotation tracers. Therefore, for determining the solar rotational profile, it is preferable to use orthogonal polynomials based on the data themselves, i.e. to use so called "discrete orthogonal polynomials" (Nikiforov et al., 2012) rather than the above mentioned continuous orthogonal polynomials.

To construct such a set of discrete orthogonal polynomials $u_{1}, u_{2} \ldots u_{k}$ using the basis $v_{1}, v_{2} \ldots v_{k}$, we utilized the Forsythe's method (Forsythe, 1957; Clenshaw, 1960). After that, we use the least square method to estimate the solar differential rotation parameters represented by the polynomial coefficients from observational data. We hereby use coronal holes as tracers of the differential rotation. The observational data have been compiled from the SDO/ AIA database for 2014 and 2015.

Note, that parameters A, B, and C in Eq. (2) represent the tracer angular velocity at the solar equator, the curvature, and curvature of the curvature of the data at the solar equator, respectively. If the data are not clustered about the equator, these parameters might be highly dependent on the polynomial used to the data. On the other hand, the coefficients of the discrete orthogonal polynomials represent the average value of the angular velocity ('rigid body' angular velocity), the average curvature and curvature of the curvature of the data, correspondingly. They are physical characteristics of the solar rotation that are independent of the degree of the fitted polynomial. Discussion on the advantage of the orthogonalization of the data in the regression analysis is in the book Bevington and Robinson (2003).

A lot of studies on differential rotation of the Sun have found a North-South asymmetry in its pattern (Zhang et al., 2015 and references therein). However, most of these studies use quadratic or biquadratic regression functions which basically excludes the possibility of asymmetry. In this paper, along with quadratic and biquadratic polynomials, we also use polynomial regression functions without omitting the odd order terms, and we compare these regression models applying the Akaike information criterion (Akaike, 1974; Feigelson and Babu, 2012).

At present, results of the coronal hole's rotation are not consistent ((Oghrapishvili et al., 2018) and references therein). Below, we have made a statistical analysis of coronal holes observations using the Forsythe's method of constructing orthogonal polynomials.

Our approach is based on the methods developed in the books by Hudson (1964),Bevington and Robinson (2003) and Seber and Lee (2003). 


\section{Methods}

\subsection{The Forsythe's method}

According to the Forsythe's method (Forsythe, 1957; Clenshaw, 1960) the system of discrete orthogonal vectors $u_{1}=u_{1}\left(x_{i}\right), u_{2}=u_{2}\left(x_{i}\right), u_{3}=u_{3}\left(x_{i}\right), \ldots, u_{k}=u_{k}\left(x_{i}\right)$, $(i=1,2, \ldots, N)$ for the linearly independent set of basis vectors

$v_{1}\left(x_{i}\right)=1, v_{2}\left(x_{i}\right)=x_{i}, \ldots, v_{k}\left(x_{i}\right)=x_{i}^{k-1}, \quad i=1,2, \ldots, N$,

is constructed as follows:

$u_{1}\left(x_{i}\right)=1, u_{2}\left(x_{i}\right)=x_{i}-\frac{\sum_{s=1}^{N} x_{s}}{N}, \quad i=1,2, \ldots, N$

$u_{j+1}\left(x_{i}\right)=\left(x_{i}-\alpha_{j+1}\right) u_{j}\left(x_{i}\right)-\beta_{j} u_{j-1}\left(x_{i}\right)$,

where

$\alpha_{j+1}=\frac{\sum_{i=1}^{N} x_{i}\left[u_{j}\left(x_{i}\right)\right]^{2}}{\sum_{i=1}^{N}\left[u_{j}\left(x_{i}\right)\right]^{2}}, \quad \beta_{j}=\frac{\sum_{i=1}^{N}\left[u_{j}\left(x_{i}\right)\right]^{2}}{\sum_{i=1}^{N}\left[u_{j-1}\left(x_{i}\right)\right]^{2}}$,

$i=1,2, \ldots, N, \quad j=3,4, \ldots, k-1$

It should be noted that discrete orthogonal polynomials can be also obtained using the modified Gram-Schmidt process (Seber and Lee, 2003; Stewart, 1998; Golub and VanGolub, 1996). This method is similar to the Forsythe's one, with the key difference that in the latter at each stage only the preceding two polynomials are involved (Hudson, 1964; Seber and Lee, 2003).

\subsection{Least square estimation. The case of the same variance}

Suppose now that we have observational data of the surface rotation velocities, i.e. measurements of angular velocity $y_{i}$ at latitude $\theta_{i}$. In this case, when the variable $x=\sin \theta$ gets only discrete values, for the regression function instead of Eqs. (2) and (4) generally we have:

$y\left(x_{i}\right)=A_{0} v_{1}\left(x_{i}\right)+A_{1} v_{2}\left(x_{i}\right)+A_{2} v_{3}\left(x_{i}\right)+\cdots+A_{k-1} v_{k}\left(x_{i}\right)$,

$i=1,2, \ldots, N$

where

$v_{1}\left(x_{i}\right)=1, \quad v_{2}\left(x_{i}\right)=x_{i}, \quad v_{3}\left(x_{i}\right)=x_{i}^{2}, \ldots \quad v_{k}\left(x_{i}\right)=x_{i}^{k-1}$.

The observational data are given by:

$y_{i}=y\left(x_{i}\right)+\epsilon_{i}, \quad i=1,2, \ldots, N$,

where $\epsilon_{i}$ corresponds to the random error of the measurement of the angular velocity $y_{i}$ at the latitude $\theta_{i}$.

In the present paper, it is assumed that we know the exact values of the $x_{i}, i=1,2, \ldots, N$. Notice that, if $v$ and $u$ are continuous variables, i.e. $v=v(x)$ and $u=u(x)$ with
$-1 \leqslant x \leqslant 1$, then application of the Gram-Schmidt procedure yields the Legendre polynomials up to constant multiplication factors (Lipschutz and Lipson, 2009).

The Gegenbauer polynomials used in Javaraiah (2011), Snodgrass and Ulrich (1990), Snodgrass and Howard (1985) result from the orthogonalization of functions representing the line-of-sight components of the rotational velocity measured by using the spectroscopic method. In principle, for applying the above-mentioned tracer motion method, the Legendre polynomials should be utilized. However, as mentioned in Section 1, the shortcomings of using such continuous polynomials still remain.

Suppose that the errors $\epsilon_{i}$ have the same known variance $\sigma^{2}$. We will consider the case where the errors $\epsilon_{i}$ have different variances $\sigma_{i}^{2}$ separately below. Considering Eq. (10), the Eq. (12) can be rewritten in matrix form as follows:

$\boldsymbol{y}=\boldsymbol{V} \boldsymbol{\alpha}_{v}+\boldsymbol{\epsilon}$,

where $\boldsymbol{y}=\left(y_{1}, y_{2} \ldots, y_{N}\right)^{T}$, is the column vector of the measurements of angular velocity $y_{i}$ (where the superscript $T$ designates the transposition), while

$\boldsymbol{\alpha}_{v}=\left(A_{0}, A_{1}, A_{2}, \ldots, A_{k-1}\right)^{T}$,

is the column vector of the parameters that are to be estimated, and

$\boldsymbol{\epsilon}=\left(\epsilon_{1}, \epsilon_{2} \ldots, \epsilon_{N}\right)^{T}$,

corresponds to the column vector of the errors, and finally

$\boldsymbol{V}=\left(\begin{array}{cccc}v_{1}\left(x_{1}\right) & v_{2}\left(x_{1}\right) & \ldots & v_{k}\left(x_{1}\right) \\ v_{1}\left(x_{2}\right) & v_{2}\left(x_{2}\right) & \ldots & v_{k}\left(x_{2}\right) \\ \cdot & \cdot & . & \cdot \\ v_{1}\left(x_{N}\right) & v_{2}\left(x_{N}\right) & \ldots & v_{k}\left(x_{N}\right)\end{array}\right)$,

is a design $N \times k$; matrix.

When the latter matrix $\boldsymbol{V}$ has a full rank (in practice the design matrix $\boldsymbol{V}$ has a full rank, as can be checked by using the observational data $\left.x_{i}, i=1,2, \ldots, N\right)$, the application of the least squares method to Eq. (10) yields the parameter estimates $\widehat{\boldsymbol{\alpha}_{v}}=\left(\widehat{A_{0}}, \widehat{A_{1}}, \widehat{A_{2}}, \ldots, \widehat{A_{k-1}}\right)^{T}$ (Hudson, 1964):

$\widehat{\boldsymbol{\alpha}_{v}}=\left(\boldsymbol{V}^{T} \boldsymbol{V}\right)^{-1} \boldsymbol{V}^{T} \boldsymbol{y}$.

The covariance matrix for the estimate $\widehat{\boldsymbol{\alpha}_{v}}$, using canonical polynomials (11), can be expressed as

$\boldsymbol{D}=\sigma^{2}\left(\boldsymbol{V}^{T} \boldsymbol{V}\right)^{-1}$.

Note that if in Eq. (18) the value of the $\sigma^{2}$ is unknown, we can use its estimate.

For non-orthogonal functions $v_{1}\left(x_{i}\right), v_{2}\left(x_{i}\right) \ldots v_{k}\left(x_{i}\right)$, the covariation matrix $\boldsymbol{D}$ given by Eq. (18) will not be diagonal because of the correlation among the non-orthogonal functions.

For the discrete observational data $x_{i}(i=1,2, \ldots, N)$, the polynomial regression function $y\left(x_{i}\right)$ can be also expressed as an algebraic sum of the discrete orthogonal polynomials obtained by the Forsythe's method. 
We thus have

$y\left(x_{i}\right)=A_{O_{0}} u_{1}\left(x_{i}\right)+A_{O_{1}} u_{2}\left(x_{i}\right)+\cdots+A_{O_{k-1}} u_{k}\left(x_{i}\right), \quad i=1,2, \ldots, N$.

The parameters $A_{O_{0}}, A_{O_{1}} \ldots A_{O_{k-1}}$ can be estimated from the observational data $\left(y_{i}, x_{i}\right) i=1,2, \ldots, N$.

In this case, instead of the Eq. (13), we have

$\boldsymbol{y}=\boldsymbol{U} \boldsymbol{\alpha}_{u}+\boldsymbol{\epsilon}$,

where

$\boldsymbol{U}=\left(\begin{array}{cccc}u_{1}\left(x_{1}\right) & u_{2}\left(x_{1}\right) & \ldots & u_{k}\left(x_{1}\right) \\ u_{1}\left(x_{2}\right) & u_{2}\left(x_{2}\right) & \ldots & u_{k}\left(x_{2}\right) \\ \cdot & \cdot & . & \cdot \\ u_{1}\left(x_{N}\right) & u_{2}\left(x_{N}\right) & \ldots & u_{k}\left(x_{N}\right)\end{array}\right)$

and

$\boldsymbol{\alpha}_{u}=\left(A_{O_{0}}, A_{O_{1}}, \ldots, A_{O_{k-1}}\right)^{T}$.

The application of the least squares method to Eq. (19) then yields the parameter estimates $\widehat{\boldsymbol{\alpha}_{u}}=\left(\widehat{A_{O_{0}}}, \widehat{A_{O_{1}}}, \ldots, \widehat{A_{O_{k-1}}}\right)^{T}$ :

$\widehat{\boldsymbol{\alpha}_{u}}=\left(\boldsymbol{U}^{T} \boldsymbol{U}\right)^{-1} \boldsymbol{U}^{T} \boldsymbol{y}$.

The covariance matrix for estimate $\widehat{\boldsymbol{\alpha}_{u}}$, using the orthogonal polynomials obtained by the Forsythe's method is given by

$\boldsymbol{D}_{O}=\sigma^{2}\left(\boldsymbol{U}^{T} \boldsymbol{U}\right)^{-1}$.

When the value of $\sigma^{2}$ is unknown, we can use its estimate.

The covariance matrix $\boldsymbol{D}_{O}$ is a diagonal matrix because of the orthogonality of the functions $u_{1}\left(x_{i}\right), u_{2}\left(x_{i}\right) \ldots$ $u_{k}\left(x_{i}\right), i=1,2, \ldots, N$ (Hudson, 1964).

Note, when design matrix $V$ does not have a full rank or $V$ is ill-conditioned, the singular value decomposition gives the regression parameter estimates (Seber and Lee, 2003). In the present work, we consider only polynomials up to degree 4. Our numerical experiments show that the standard least square method and the singular value decomposition give the exact same parameter estimates in all performed calculations.

\subsection{Weighted least square estimation. The case of the different variances.}

In case the errors $\epsilon_{i}$ have different known variances $\sigma_{i}^{2}=\frac{\sigma^{2}}{w_{i}}$, where $w_{i}$ is the known weight of the $i$-th observation, the parameter estimates are obtained using weighted least square method.

\section{Results and discussion}

\subsection{Comparing different polynomials}

The solar differential rotation is expressed in terms of polynomials. The conditional equations use discrete quadratic polynomials (Newton and Nunn, 1951)
$y\left(x_{i}\right)=A_{0}+A_{2} x_{i}^{2}, \quad i=1,2, \ldots, N$,

or discrete biquadratic polynomials (Howard and Harvey, 1970)

$y\left(x_{i}\right)=A_{0}+A_{2} x_{i}^{2}+A_{4} x_{i}^{4}, \quad i=1,2, \ldots, N$.

Note that the coefficients $A_{0}, A_{2}$ and $A_{4}$ are usually referred to as $A, B$ and $C$, respectively. These equations are obtained empirically and imply that the differential rotational law is the same in both hemispheres. However, there is evidence (Zhang et al., 2015 and references therein) of North-South asymmetries in the solar differential rotation pattern. Therefore, it is relevant to compare the quality of a set of statistical models to each other using an appropriate statistical criterion. One of the more widely used modern criteria is the Akaike information criterion (AIC) (Akaike, 1974; Feigelson and Babu, 2012) which selects the best model from a set of considered models. In estimating the amount of information lost by a model, AIC deals with the tradeoff between the goodness of fit of the model and the simplicity of the model. The basic equation of this criterion for least squares regression-type analyses for normally distributed errors is

$A I C=N \ln (\hat{S} / N)+2 K$,

where $\hat{S}$ corresponds to the residual of the sum of squares, while $N$ is the sample size, ad $K$ denotes the number of model parameters. The estimated variance must be included in the parameter count. According to this criterion, the best model in a set of models must have the lowest AIC score (Burnham and Anderson, 2002).

To check the robustness of the proposed approach we consider the problems of estimating the solar rotational parameters for coronal holes based on the SDO/AIA data assuming the same variance in measurement errors $\left(w_{i}=1, i=1,2, \ldots, N\right)$ (Oghrapishvili et al., 2018). The heliographic coordinates of the centroids of the tracers on consecutive images of the solar surface have been calculated. This enabled us to determine the sidereal angular velocity $\omega\left(\theta_{i}\right)$ of the specified $i$-th tracer on its corresponding latitude $\theta_{i}$. The data were analyzed using the following five types of the basis polynomials of discrete variable $x_{i}$ $\left(x_{i}=\sin \theta_{i}\right)$ :

(1) Canonical quadratic polynomials, no linear term

$$
\begin{aligned}
& v_{1}\left(x_{i}\right)=1, \quad v_{2}\left(x_{i}\right)=x_{i}^{2} ; \quad y\left(x_{i}\right)=A_{0}+A_{2} x_{i}^{2} \\
& i=1,2, \ldots, N .
\end{aligned}
$$

(2) Canonical bi-quadratic polynomials

$$
\begin{aligned}
& v_{1}\left(x_{i}\right)=1, v_{2}\left(x_{i}\right)=x_{i}^{2}, \quad v_{3}\left(x_{i}\right)=x_{i}^{4} ; \\
& y\left(x_{i}\right)=A_{0}+A_{2} x_{i}^{2}+A_{4} x_{i}^{4}, \quad i=1,2, \ldots, N .
\end{aligned}
$$


(3) Canonical polynomials of degree 4

$$
\begin{aligned}
& v_{1}\left(x_{i}\right)=1, \quad v_{2}\left(x_{i}\right)=x_{i}, \quad v_{3}\left(x_{i}\right)=x_{i}^{2}, \\
& v_{4}\left(x_{i}\right)=x_{i}^{3}, \quad v_{5}\left(x_{i}\right)=x_{i}^{4} ; \\
& y\left(x_{i}\right)=A_{0}+A_{1} x_{i}+A_{2} x_{i}^{2}+A_{3} x_{i}^{3}+A_{4} x_{i}^{4}, \\
& i=1,2, \ldots, N .
\end{aligned}
$$

(4) Legendre polynomials of degree 4

$$
\begin{aligned}
& v_{1}(x)=1, \quad v_{2}(x)=x, \quad v_{3}(x)=x^{2}, \\
& v_{4}(x)=x^{3}, \quad v_{5}(x)=x^{4} \\
& y\left(x_{i}\right)=A_{L_{0}}+A_{L_{1}} x_{i} \\
& +A_{L_{2}}\left(x_{i}^{2}-\frac{1}{3}\right) \\
& +A_{L_{3}}\left(x_{i}^{3}-\frac{3}{5} x_{i}\right) \\
& +A_{L_{4}}\left(x_{i}^{4}-\frac{30}{35} x_{i}^{2}+\frac{3}{35}\right) \\
& i=1,2, \ldots, N .
\end{aligned}
$$

(5) Discrete orthogonal polynomials of degree 4

$$
\begin{aligned}
& v_{1}\left(x_{i}\right)=1, \quad v_{2}\left(x_{i}\right)=x_{i}, \quad v_{3}\left(x_{i}\right)=x_{i}^{2}, \\
& v_{4}\left(x_{i}\right)=x_{i}^{3}, \quad v_{5}\left(x_{i}\right)=x_{i}^{4} ; \\
& y\left(x_{i}\right)=A_{O_{0}} u_{1}\left(x_{i}\right)+A_{O_{1}} u_{2}\left(x_{i}\right) \\
& +A_{O_{2}} u_{3}\left(x_{i}\right)+A_{O_{3}} u_{4}\left(x_{i}\right)+A_{O_{4}} u_{5}\left(x_{i}\right), \\
& i=1,2, \ldots, N,
\end{aligned}
$$

where $u_{1}\left(x_{i}\right), u_{2}\left(x_{i}\right), u_{3}\left(x_{i}\right), u_{4}\left(x_{i}\right)$, and $u_{5}\left(x_{i}\right)$ can be obtained using both the Forsythe's method and the modified Gram-Schmidt procedure (Golub and VanGolub, 1996; Stewart, 1998) as well. Farther numerical calculations showed the same results for both approaches.

\subsection{Data reduction and results}

The rotation of coronal holes $(\mathrm{CHs})$ was studied using the data from the coronal hole catalog http://www.solspanet.eu compiled within the framework of the project SOLSPLANET (Solar and Space Weather Network of Excellence) for 2014 and 2015. CHs are identified using the Spatial Possibilistic Clustering Algorithm (SPoCA), which yields the locations with uncertainties and other characteristics of solar $\mathrm{CHs}$ on SDO/AIA $193 A^{\circ}$ wavelength images (Verbeeck et al., 2014). Instead of calculating average angular velocities of each coronal hole centroid and then grouping them in latitudinal bins for calculating average rotation rates of corresponding latitudes, we computed and compiled instant rotation rates of centroids and their corresponding heliographic coordinates in one matrix for further processing as described in detail in (Oghrapishvili et al., 2018). Even the unfiltered data clearly showed the differential rotation of coronal holes. To avoid different projection effects that affect the data, a \pm 20 degrees wide longitudinal zone was selected to proceed with the study. It was examined using different methods of data filtering. It was found that the filtration using local medians of the data with a constant threshold ( $30 \%$ of the targeted value) is the most acceptable approach because it is not biased towards a predefined notion of an expected result.

Our calculations show that the uncertainties of the latitude of the geometric center of the coronal hole in the stripe of width \pm 60 degrees are less than 0.45 degree and are about 8 degrees in the circumpolar zones (see Fig. 1). Monte Carlo simulations show that in the latitudinal zone of \pm 60 degrees, the uncertainties of the latitudes of the geometric center of coronal hole make changes in the least square parameter estimates with a value of not more than one hundredth. It can be seen below that this is significantly less than the influence of the choice of the polynomial types on the parameter estimates in the curve fitting process. Thus, these uncertainties can be neglected. Only latitudinal zone data of \pm 60 degrees are used in the work. It gives 2883 sidereal angular velocity measurements with equal weights $w_{i}=1, i=1,2, \ldots, N, N=2883$. The obtained results from the application of the least square methods to estimate the solar differential rotation parameters (coefficients) shown above do not require knowledge of the distribution functions of the errors (residuals) $\epsilon_{i}, i=1,2, \ldots, N$. As a matter of fact, it is sufficient to know the information about the variances of the errors. If we assume that the errors $\epsilon$ are distributed normally with zero mean and the same known variance $\sigma^{2}$, then it can be shown that the estimates of the coefficients also will be distributed normally with known variances defined by the covariance matrix (Hudson, 1964).

We analyzed the coronal hole data for the measurement of the solar differential rotation. In Fig. 2 panel A represents the normal cumulative distribution function (cdf) with the estimated variance $\sigma$ and the empirical cdf of residuals $\epsilon_{i}$ for the polynomials of degree 4. Panel B shows the difference between normal cdf and the empirical one. As is seen from this figure the distribution of $\epsilon_{i}$ can be very well approximated by a normal pdf.

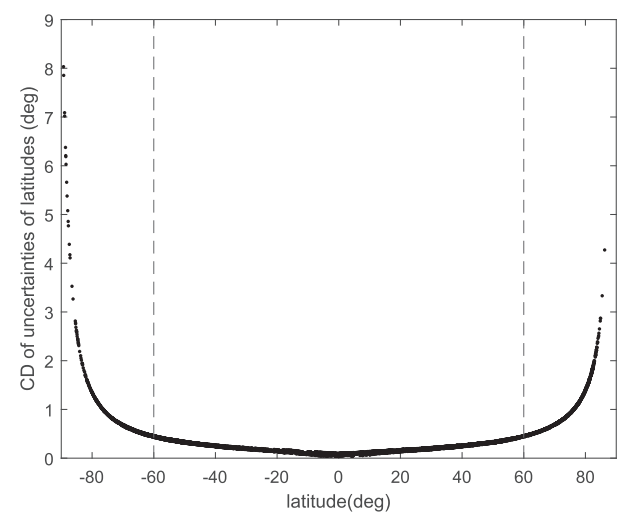

Fig. 1. Latitudinal distribution of the standard deviations (SD) of $\mathrm{CH}$ centroid uncertainties. Vertical discrete lines denote the latitudinal data zone of the sample data. 

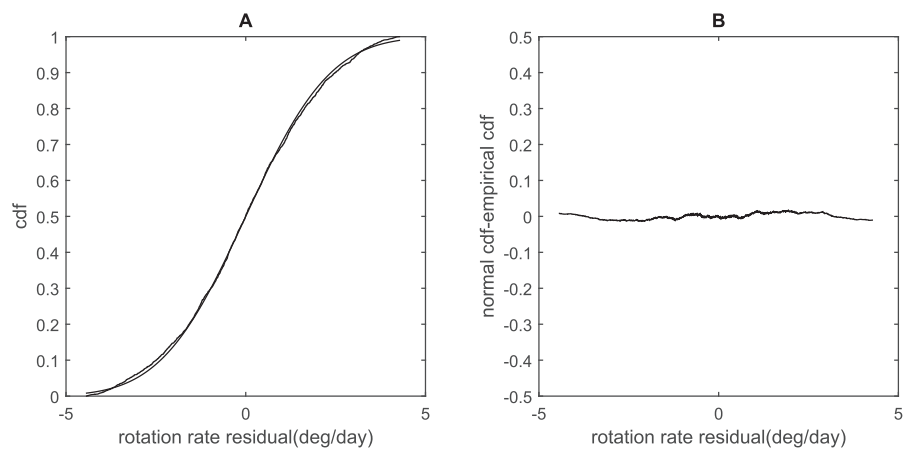

Fig. 2. (A) The normal cdf (smooth line) and the empirical cdf of the residuals (broken line). (B) The difference between the normal cdf and the empirical cdf of residuals vs rotation rate residuals.

Using our approach, we construct the confidence intervals for the regression function (see below). Results of the application of the least square method for polynomial fitting and the corresponding AIC scores are presented in Table 1.

In Fig. 3 the profiles of the latitudinal distribution of the rotation rates of magnetic features at the photospheric level (Snodgrass and Ulrich, 1990), coronal bright points (Sudar et al., 2015) and coronal holes are given. The results for the bright points show lower values than those of photospheric magnetic features for lower latitude (within about \pm 30 degrees). Our results lie between them. For higher latitudes, our results are steeper, while bright coronal points and photospheric magnetic features almost follow each other with some lower values for coronal bright points. On the whole, it can be seen that our results show lower values than the photospheric magnetic features at all latitudes. This can be caused by the delaying effect of the chromospheric layer, which is penetrated by a magnetic field, the holder of coronal holes (in the corona, the magnetic pressure in the coronal holes exceeds the kinetic pressure, and the magnetic field determines the behavior of the coronal hole, but this should not be the case in the underlying layers, in the chromosome and photosphere).

Fig. 3 definitely shows a pattern of differential rotation. It should be noted that applications of all mentioned polynomials of degree 4 give the same fitting curve. This is due to the fact that these polynomials can be obtained from each other by regrouping the terms (Bevington and Robinson, 2003; Hudson, 1964). On the other hand, the coefficients for the different basis polynomials will differ. In Table 1, we present estimates of the coefficients and their standard deviations for the mentioned polynomials obtained using the application of the least square method. To check the different polynomial models, we compare their covariance matrices. For each type of polynomials (the type is indicating by the subscript) the corresponding covariance matrices are shown in the following equations:

$$
\begin{aligned}
\boldsymbol{D}_{C Q} & =\left(\begin{array}{ccc}
0.0024 & -0.0054 \\
-0.0054 & 0.0229
\end{array}\right), \\
\boldsymbol{D}_{C B} & =\left(\begin{array}{cccc}
0.0033 & -0.0194 & 0.0229 \\
-0.0194 & 0.2575 & -0.3823 \\
0.0229 & -0.3823 & 0.6228
\end{array}\right), \\
\boldsymbol{D}_{C 4} & =\left(\begin{array}{ccccc}
0.0033 & 0.0007 & -0.0198 & -0.0008 & 0.0236 \\
0.0007 & 0.0370 & -0.0116 & -0.0696 & 0.0114 \\
-0.0198 & -0.0116 & 0.2698 & 0.0074 & -0.4040 \\
-0.0008 & 0.0696 & 0.0074 & 0.01537 & 0.0080 \\
0.0236 & 0.0114 & -0.4040 & 0.0080 & 0.6636
\end{array}\right),
\end{aligned}
$$

$$
\boldsymbol{D}_{L 4}=\left(\begin{array}{lllll}
0.0022 & 0.0010 & 0.0079 & 0.0032 & 0.0217 \\
0.0010 & 0.0088 & 0.0068 & 0.0226 & 0.0162 \\
0.0079 & 0.0068 & 0.0648 & 0.0142 & 0.1648 \\
0.0032 & 0.0226 & 0.0142 & 0.1537 & 0.0080 \\
0.0217 & 0.0162 & 0.1648 & 0.0080 & 0.6636
\end{array}\right)
$$

\begin{tabular}{|c|c|c|c|c|c|c|}
\hline Type & $\begin{array}{c}\widehat{A}_{T_{0}} \\
{\left[\begin{array}{c}{ }^{-1} \mathrm{day}^{-1}\end{array}\right]}\end{array}$ & $\begin{array}{c}\widehat{A}_{T_{1}} \\
{\left[\begin{array}{c}{ }^{\circ} \mathrm{day}^{-1}\end{array}\right]}\end{array}$ & $\begin{array}{c}\widehat{A}_{T_{2}} \\
{\left[\begin{array}{c}{ }^{\circ} \mathrm{day}^{-1}\end{array}\right]}\end{array}$ & {$\left[\begin{array}{c}\widehat{A}_{T_{3}} \\
{\left[\begin{array}{c}\mathrm{day}^{-1}\end{array}\right]}\end{array}\right.$} & $\begin{array}{c}\widehat{A}_{T_{4}} \\
{\left[\begin{array}{c}{ }^{\circ} \mathrm{day}^{-1}\end{array}\right]}\end{array}$ & $\overline{\mathrm{AIC}}$ \\
\hline CQ & $14.67 \pm 0.05$ & & $-5.13 \pm 0.15$ & & & 3567 \\
\hline $\mathrm{CB}$ & $14.57 \pm 0.05$ & & $-3.45 \pm 0.51$ & & $-2.74 \pm 0.79$ & 3557 \\
\hline L4 & $12.88 \pm 0.05$ & $0.01 \pm 0.09$ & $-5.81 \pm 0.25$ & $0.83 \pm 0.39$ & $-3.00 \pm 0.81$ & 3554 \\
\hline $\mathrm{O} 4$ & $13.47 \pm 0.03$ & $0.17 \pm 0.07$ & $-5.14 \pm 0.15$ & $0.87 \pm 0.39$ & $-3.00 \pm 0.81$ & 3554 \\
\hline
\end{tabular}

Table 1

Parameter estimates of the solar rotation profile for four different types of basis polynomials. 

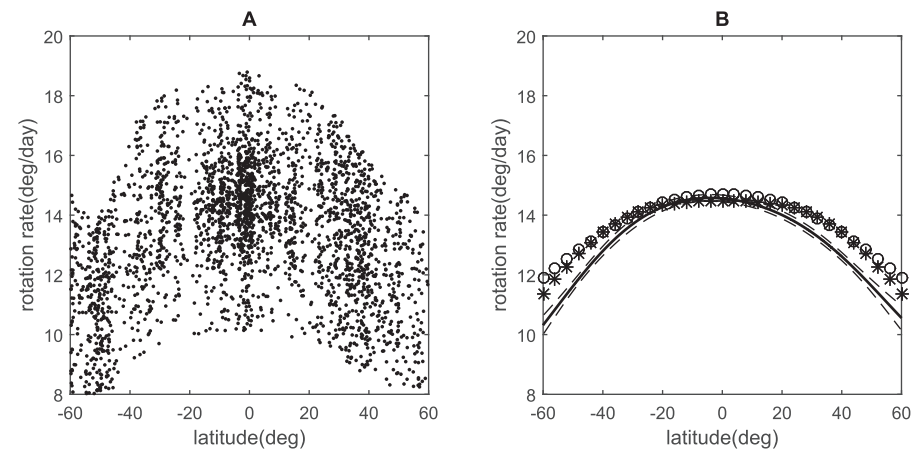

Fig. 3. (A) Sample data studied (dots). (B) Differential rotation profiles for photospheric magnetic features (circles), coronal bright points (asterix) and coronal holes (soid line) using orthogonal polynomials of degree 4 with appropriate $95 \%$ level confidence intervals along the ordinate axis (dashed lines).

$$
\boldsymbol{D}_{O 4}=\left(\begin{array}{lllll}
0.0012 & 0.0000 & 0.0000 & 0.0000 & 0.0000 \\
0.0000 & 0.0051 & 0.0000 & 0.0000 & 0.0000 \\
0.0000 & 0.0000 & 0.0230 & 0.0000 & 0.0000 \\
0.0000 & 0.0000 & 0.0000 & 0.1536 & 0.0000 \\
0.0000 & 0.0000 & 0.0000 & 0.0000 & 0.6636
\end{array}\right),
$$

where $\boldsymbol{D}_{C Q}, \boldsymbol{D}_{C B}, \boldsymbol{D}_{C 4}, \boldsymbol{D}_{L 4}$, and $\boldsymbol{D}_{O 4}$ are the covariance matrices using canonical quadratic polynomials (CQ), canonical biquadratic polynomials $(\mathrm{CB})$, canonical polynomials of degree $4(\mathrm{C} 4)$, Legendre polynomials of degree 4 (L4) and orthogonal polynomials of degree 4 (O4) correspondingly for the whole $(N=2883)$ data set.

The model without correlated errors (i.e. without 'crosstalk') among the estimated coefficients should have a diagonal form covariance matrix. Computational equations for covariance matrices are given above (Eqs. (18) and (24)). For Legendre polynomials, the covariance matrix is obtained analogous to Eqs. (18) and (24). In this case, the design matrix is constructed using Legendre polynomials of degree 4.

It is seen from Eqs. (33)-(37) that only the covariance matrix obtained using orthogonal polynomials has a diagonal form. The coefficients of these polynomials are estimated by means of the Forsythe's method. We utilized this fact to construct confidence intervals for the regression function (32). For estimating the regression function and its variance we have

$$
\begin{aligned}
\widehat{y}\left(x_{i}\right)= & \widehat{A}_{O_{0}} u_{1}\left(x_{i}\right)+\widehat{A}_{O_{1}} u_{2}\left(x_{i}\right)+\widehat{A}_{O_{2}} u_{3}\left(x_{i}\right)+\widehat{A}_{O_{3}} u_{4}\left(x_{i}\right) \\
& +\widehat{A}_{O_{4}} u_{5}\left(x_{i}\right), \quad i=1,2, \ldots, N,
\end{aligned}
$$

and

$$
\begin{aligned}
\sigma_{\widehat{y}\left(x_{i}\right)}^{2}= & {\left[u_{1}\left(x_{i}\right)\right]^{2} \sigma_{\widehat{A} O_{0}}^{2}+\left[u_{2}\left(x_{i}\right)\right]^{2} \sigma_{\widehat{A}}^{2}+\left[u_{3}\left(x_{i}\right)\right]^{2} \sigma_{\widehat{A}}^{2} } \\
& +\left[u_{4}\left(x_{i}\right)\right]^{2} \sigma_{\widehat{A} O_{O_{3}}}^{2}+\left[u_{5}\left(x_{i}\right)\right]^{2} \sigma_{\widehat{A}_{O_{4}}}^{2}, \quad i=1,2, \ldots, N,
\end{aligned}
$$

where $u_{1}\left(x_{i}\right), u_{2}\left(x_{i}\right), u_{3}\left(x_{i}\right), u_{4}\left(x_{i}\right)$ and $u_{5}\left(x_{i}\right)$ are obtained by applying the Forsythe's method and $\widehat{A}_{O_{0}}, \widehat{A}_{O_{1}}, \widehat{A}_{O_{2}}, \widehat{A}_{O_{3}}$ and
$\widehat{A}_{O_{4}}$, and the variances $\sigma_{\widehat{A}_{O_{0}}}^{2}, \sigma_{\widehat{A}_{O_{1}}}^{2}, \sigma_{\widehat{A}_{O_{2}}}^{2}, \sigma_{\widehat{A}_{O_{3}}}^{2}$, and $\sigma_{\widehat{A}_{O_{4}}}^{2}$ are obtained using the least squares method (20)-(24).

Note that the covariance terms in Eq. (39) vanish because the covariance matrix $D_{O 4}$ is diagonal. The estimate $\widehat{y}\left(x_{i}\right)$ will be normally distributed around the $y\left(x_{i}\right)$ with the variance $\sigma_{\hat{y}\left(x_{i}\right)}^{2}$. An estimate of the variance $\sigma_{\hat{y}\left(x_{i}\right)}^{2}$ can be obtained from Eq. (39) by substituting the variances on the right hand side of the equation by their estimates.

The confidence intervals for the estimates of the regression function $\widehat{y}\left(x_{i}\right)$ can be constructed in a standard way (Feigelson and Babu, 2012):

$$
\begin{aligned}
& 95 \% \text { CI for } \widehat{y}\left(x_{i}\right) \\
& \quad=\left(\widehat{y}\left(x_{i}\right)-1.96 \cdot \widehat{\sigma}_{\widehat{y}\left(x_{i}\right)}, \widehat{y}\left(x_{i}\right)+1.96 \cdot \widehat{\sigma}_{\widehat{y}\left(x_{i}\right)}\right), \quad i=1,2, \ldots, N .
\end{aligned}
$$

In Table 1 the minimum (i.e. best) AIC score 3554 is obtained by the polynomials of degree 4 . As we mentioned before, all the considered polynomials (C4, L4 and O4) yield the same fitting curve.

\section{Summary and conclusion}

To construct the discrete orthogonal polynomials in the modeling of the solar differential rotation, we used the Forsythe's method (Forsythe, 1957; Clenshaw, 1960). and the modified Gram-Schmidt procedure (Golub and VanGolub, 1996; Stewart, 1998; Seber and Lee, 2003).

Using the least square method for the rotation parameter estimates implies that we know the exact latitudes of the centroids. But the uncertainties of the observed latitudes differ from zero and is less than 0.45 degrees (Fig. 1). Statistical analysis showed that these uncertainties can be neglected in the estimation of the least squares of $\mathrm{CHs}$ rotation parameters.

According to the results of the testing with the Akaike Information Criterion (Akaike, 1974; Feigelson and Babu, 2012), we can state that the best models among the considered canonical quadratic, canonical biquadratic, canonical polynomials of degree 4, discrete orthogonal polynomials of degree 4 and Legendre polynomials 
of degree 4, are the three models with polynomials of degree 4.

The coefficients $A_{0}, A_{1}, A_{2}$ and so forth, represent the tracers angular velocity at the solar equator, the slope of the angular velocity data at the equator, the curvature of the data at the solar equator and so forth, respectively. If the data are not clustered about the equator, these coefficients might be highly dependent on the polynomial used to fit the data.

The coefficients of the discrete orthogonal polynomials $A_{O_{0}}, A_{O_{1}}, A_{O_{2}}$ etc., represent the average value of the angular velocity ('rigid body' angular velocity), the average value of the slope of the angular velocity data, the average curvature of the data and so forth, correspondingly. They are physical characteristics of the data that are independent of the degree of the fitted polynomial (Bevington and Robinson, 2003).

The estimates of the coefficients of the orthogonal polynomials of degree 4 are close to the coefficient estimates of the Legendre polynomials of degree 4 but still differ. Using the Legendre polynomials for discrete data gives nonorthogonal basis vectors (the covariance matrix is not diagonal), and the resulting coefficients depend on the degree of the polynomial. Therefore, it is impossible to compare the results even for the same sample data, but with a different order of polynomials. This disadvantage is eliminated by using discrete orthogonal polynomials obtained using the Forsythe's method of orthogonalization.

Comparison of the canonical, orthogonal and Legendre polynomial approaches shows that they give the same fitting curve describing the differential pattern of the Solar rotation. The coefficients of these approaches are linearly interrelated.

Hence, the orthogonal polynomial approach has advantages. For instance, it simplifies the construction of the confidence intervals for the fitting curve (38)-(40) and the estimates of the coefficients are free from 'cross talk', i.e. they are not correlated.

Therefore, we suggest that the solar differential rotation can be best modeled using the discrete orthogonal polynomials of degree 4 . As a matter of fact, the numerical experiments described in the present paper indicate that the model yields results between the over fitting and under fitting of sample data.

Careful analysis of the coefficient estimates of the discrete orthogonal polynomials of degree 4 indicates that the coefficients of the odd order terms are small, viz. $A_{O 1}=0.17 \pm 0.07$ and $A_{O 3}=0.87 \pm 0.39$. However, they are significantly different from zero at the level 0.05 . This can be interpreted as evidence of the N-S asymmetry in the solar differential rotation.

We finally remark that the proposed approach could be successfully applied for investigating the solar differential rotation using other tracers as well.

\section{Declaration of Competing Interest}

The authors declare that they have no known competing financial interests or personal relationships that could have appeared to influence the work reported in this paper.

\section{Acknowledgements}

The work was supported by European FP7-PEOPLE2010-IRSES-269299 project - SOLSPANET. Work was supported by Shota Rustaveli National Science Foundation grants N217188, DI-2016-52 and FR17_609. B.M.S. acknowledges the support by the Austrian Fonds zur Foerderung der Wissenschaftlichen Forschung within the projects P25640-N27, S11606-N16 and Leverhulme Trust grant IN-2014-016. A.K. was supported by NASA grant NNX14AB70G. The research was partially supported by the NASA Grants NNX14AB68G and NNX16AP05H.

The AIA data used here are courtesy of SDO (NASA) and the AIA consortium.

The authors would like to thank the unknown referee for the useful comments and suggestions, which were taken into account when revising the original paper.

\section{References}

Akaike, H., 1974. A new look at the statistical model identification. IEEE Trans. Autom. Control AC-19, 716-723.

Beck, J.G., 2000. A comparison of differential rotation measurements (Invited Review). Sol. Phys. 191, 47-70. https://doi.org/10.1023/ A:1005226402796.

Bevington, P.R., Robinson, D.K., 2003. Data reduction and error analysis for the physical sciences.

Burnham, K.P., Anderson, D.R., 2002. Model Selection and Multimodel Inference: A Practical Information-Theoretic Approach, 67. https:// doi.org/10.1007/b97636.

Clenshaw, C.W., 1960. Curve fitting with a digital computer. Comput. J. 2, 170-173. https://doi.org/10.1093/comjnl/2.4.170, arXiv:http://oup. prod.sis.lan/comjnl/article-pdf/2/4/170/1273963/020170.pdf.

Feigelson, E.D., Babu, G.J., 2012. Modern Statistical Methods for Astronomy.

Forsythe, G.E., 1957. Generation and use of orthogonal polynomials for data-fitting with a digital computer. J. Soc. Ind. Appl. Math. 5, 74-88, URL: http://www.jstor.org/stable/2098723.

Gigolashvili, M.S., Japaridze, D.R., Mdzinarishvili, T.G., 2011. Statistical study of the north-south asymmetry of the solar differential rotation based on various solar structures during 1966-1985. Astrophysics 54, 593-602. https://doi.org/10.1007/s10511-011-9208-3.

Gigolashvili, M.S., Japaridze, D.R., Mdzinarishvili, T.G., 2013. Investigation of $\mathrm{N}-\mathrm{S}$ asymmetry of solar differential rotation by various patterns for solar cycles 20 and 21. Adv. Space Res. 52, 2122-2131. https://doi.org/10.1016/j.asr.2013.09.032.

Gigolashvili, M.S., Japaridze, D.R., Mdzinarishvili, T.G., Chargeishvili, B.B., 2005. N S asymmetry in the solar differential rotation during 1957 1993. Sol. Phys. 227, 27-38. https://doi.org/10.1007/s11207-0051214-3.

Gigolashvili, M.S., Japaridze, D.R., Mdzinarishvili, T.G., Chargeishvili, B.B., Kukhianidze, V.J., 2007. Investigation of the N S asymmetry of the differential rotation of H $\alpha$ filaments and large-scale magnetic 
elements. Adv. Space Res. 40, 976-980. https://doi.org/10.1016/j. asr.2007.03.103.

Gigolashvili, M.S., Mdzinarishvili, T.G., Japaridze, D.R., Chargeishvili, B.B., 2003. N-S asymmetry in the differential rotation of the sun and its variation with the solar activity cycles. New Astron. 8, 529-536. https://doi.org/10.1016/S1384-1076(03)00046-0.

Golub, G.H., Van Loan, C.F., 1996. Matrix Computations, third ed. The Johns Hopkins University Press.

Howard, R., Harvey, J., 1970. Spectroscopic determinations of solar rotation. Sol. Phys. 12, 23-51. https://doi.org/10.1007/ BF02276562.

Hudson, D.J., 1964. Lectures on elementary statistics and probability. In: Lectures on Elementary Statistics and Probability. Geneva: [CERN, European Organization for Nuclear Research]. URL: http:// www.worldcat.org/title/lectures-on-elementary-statistics-and-probability/oclc/21051013.

Javaraiah, J., 2011. Quasi 9 and 30-40 days periodicities in the solar differential rotation. Adv. Space Res. 48, 1032-1040. https://doi.org/ 10.1016/j.asr.2011.05.004, arXiv: 1105.4035.

Kosovichev, A.G., 1988. Determining the internal rotation of the Sun from the frequency splitting of acoustic modes. Bull. Crimean Astrophys. Observatory 80, 167.

Lipschutz, S., Lipson, V.L., 2009. Linear algebra. In: Linear Algebra. McGraw-Hill. URL: http://www.astronomia.edu.uy/progs/algebra/ Linear_Algebra,_4th_Edition_(2009)Lipschutz-Lipson.pdf.

Newton, H.W., Nunn, M.L., 1951. The Sun's rotation derived from sunspots 1934-1944 and additional results. Mon. Not. R. Astron. Soc. 111, 413. https://doi.org/10.1093/mnras/111.4.413.

Nikiforov, A.F., Suslov, S.K., Uvarov, V.B., 2012. Classical orthogonal polynomials of a discrete variable. In: Classical Orthogonal Polynomials of a Discrete Variable. Springer, Berlin Heidelberg. URL: https://www.abebooks.com/servlet/BookDetailsPL?bi=20055662178 \&searchurl=sortby $\% 3 \mathrm{D} 17 \% 26 \mathrm{an} \% 3 \mathrm{Darnold} \% 2 \mathrm{Bf} \% 2 \mathrm{Bnikiforov} \%$ 2Band $\% 2$ Bsergei $\% 2 B k \% 2 B$ suslov\&cm_sp=snippet__-srp1-_title1.

Oghrapishvili, N.B., Bagashvili, S.R., Maghradze, D.A., Gachechiladze, T.Z., Japaridze, D.R., Shergelashvili, B.M., Mdzinarishvili, T.G., Chargeishvili, B.B., 2018. Study of the solar coronal hole rotation.
Adv. Space Res. 61, 3039-3050. https://doi.org/10.1016/j. asr.2018.03.033.

Paternò, L., 2010. The solar differential rotation: a historical view. Astrophys. Space Sci. 328, 269-277. https://doi.org/10.1007/s10509009-0218-0.

Schou, J., Antia, H.M., Basu, S., Bogart, R.S., Bush, R.I., Chitre, S.M., Christensen-Dalsgaard, J., Di Mauro, M.P., Dziembowski, W.A., EffDarwich, A., Gough, D.O., Haber, D.A., Hoeksema, J.T., Howe, R., Korzennik, S.G., Kosovichev, A.G., Larsen, R.M., Pijpers, F.P., Scherrer, P.H., Sekii, T., Tarbell, T.D., Title, A.M., Thompson, M.J., Toomre, J., 1998. Helioseismic studies of differential rotation in the solar envelope by the solar oscillations investigation using the Michelson Doppler Imager. Astrophys. J. 505, 390-417. https://doi.org/10.1086/306146.

Seber, G.A., Lee, A.J., 2003. Linear Regression Analysis.

Snodgrass, H.B., Howard, R., 1985. Torsional oscillations of low mode. Sol. Phys. 95, 221-228. https://doi.org/10.1007/BF00152399.

Snodgrass, H.B., Ulrich, R.K., 1990. Rotation of Doppler features in the solar photosphere. Astrophys. J. 351, 309-316. https://doi.org/ 10.1086/168467.

Stewart, G.W., 1998. Matrix Algorithms: Volume 1, Basic Decompositions. first ed., SIAM.

Sudar, D., Skokić, I., Brajša, R., Saar, S.H., 2015. Steps towards a high precision solar rotation profile: results from SDO/AIA coronal bright point data. Astronom. Astrophys. 575, A63. https://doi.org/10.1051/ 0004-6361/201424929, arXiv:1501.01285.

Verbeeck, C., Delouille, V., Mampaey, B., De Visscher, R., 2014. The SPoCA-suite: Software for extraction, characterization, and tracking of active regions and coronal holes on EUV images. Astronom. Astrophys. 561, A29. https://doi.org/10.1051/0004-6361/201321243.

Yoshimura, H., 1996. Solar Dynamo Driven by Global Convection and Differential Rotation and the Magneto-Thermal Pulsation of the Sun. In: Uchida, Y., Kosugi, T., Hudson, H.S. (Eds.), IAU Colloq. 153: Magnetodynamic Phenomena in the Solar Atmosphere - Prototypes of Stellar Magnetic Activity, p. 345.

Zhang, L., Mursula, K., Usoskin, I., 2015. Solar surface rotation: N-S asymmetry and recent speed-up. Astronom. Astrophys. 575, L2. https://doi.org/10.1051/0004-6361/201425169, arXiv:1501.02698. 